\title{
The role of 3D echocardiographic imaging in the differential diagnosis of an atypical left atrial myxoma
}

\author{
Domenico Galzerano1, Claudio Pragliola1, Mohammed Al Admawi1, Mario Mallardo², \\ Sara Di Michele3 ${ }^{3}$ Carlo Gaudio 3 \\ 1 The Heart Center, King Faisal Specialist Hospital \& Research Center, Riyadh, Saudi Arabia \\ 2 Cardiology Unit, S. Gennaro Hospital, Naples, Italy \\ 3 Department of Cardiovascular, Respiratory, Nephrological, Anaesthesiological and Geriatric Sciences, \\ "La Sapienza" University of Rome, Italy
}

\begin{abstract}
We describe a case of a left atrial myxoma atypical for its anatomical features and site of attachment. Although an initial multimodality imaging was performed, the diagnosis of myxoma was possible only by three-dimensional echocardiography (3DE) which was able to identify the peduncle and the attachment at the base of the interatrial septum, close to the origin of right inferior pulmonary vein. In fact, the 3DE can
\end{abstract}

Corresponding author: Domenico Galzerano, King Faisal Specialist Hospital \& Research Center, P.0. Box 3354, Riyadh 11211 MBC, Saudi Arabia. Tel. +966.014647272 Ext: 34949 - Mobile: $+966.539037762 ;+39.3284157343$. E-mail: domenicogalzerano@libero.it

Key words: Three-dimensional echocardiography; cardiac myxoma; nuclear magnetic resonance.

Contributions: DG, clinical data collection, echocardiographic imaging interpretation, manuscript writing, reviewing and approval of final version; CP, surgery on patients, intraoperative pictures shooting, manuscript writing, reviewing and approval of final version; MAA, interpretation of MRI and CT scan imaging, manuscript writing, reviewing and approval of final version; MM, echocardiographic imaging interpretation, manuscript writing, reviewing and approval of final version; SDM, echocardiographic and radiological interpretation, manuscript reviewing and approval of final version; CG, clinical results interpretation, manuscript writing, reviewing and approval of final version. All the authors equally contributed to this work.

Conflict of interest: The authors declare they have no conflict of interest.

Acknowledgements: We would like to thank Joanne London for her professional technical assistance and kind revision of the manuscript.

Received for publication: 27 December 2017

Accepted for publication: 18 July 2018

CCopyright D. Galzerano et al., 2018

Tipografia PI-ME Editrice, Italy

Monaldi Archives for Chest Disease 2018; 88:906

doi: 10.4081/monaldi.2018.906

This article is distributed under the terms of the Creative Commons Attribution Noncommercial License (by-nc 4.0) which permits any noncommercial use, distribution, and reproduction in any medium, provided the original author(s) and source are credited. electronically section the structures and obtain unique planes useful in visualizing correctly the anatomical features of the myxomas and as a result, it facilitates the surgical decision planning. Even the anatomical appearance was uncommon at surgery and the diagnosis could be confirmed only by pathology. This case highlights the diagnostic ability of the 3DE in similar challenging scenarios.

\section{Introduction}

The majority of cardiac myxomas manifest as endocavitary gelatinous masses that arise adjacent to the fossa ovalis in the left atrium. They typically have a peduncle but can also arise in a sessile fashion [1-5]. The majority (75-80\%) are located within the left atrium, characteristically originating from the mid-portion of the atrial septum by a narrow stalk; 15-20\% originate within the right atrium [1-5].

Detection by imaging of the above anatomical findings easily lead to the diagnosis [3-7]. We describe a case of an unusual left atrial (LA) myxoma whose peduncle and attachment to the base of interatrial septum (IAS) was detected only by using the two-dimensional (2D) planes as generated by three-dimensional echocardiography (3DE).

\section{Case Report}

A 57-year-old, hypertensive, diabetic, female patient with a history of previous cerebrovascular accident (CVA), in sinus rhythm, underwent a transthoracic echocardiography (TTE) to detect the source of embolism following a newly diagnosed CVA. The exam showed a large mobile mass in the left atrium (size $1.7 \times 3 \mathrm{~cm}$ ), with irregular shape and borders (Figure 1A), and with an inhomogeneous echo-structure. The mass seemed to originate from the right inferior pulmonary vein (RIPV) or from the basal IAS. Left atrium was normal in size. No other abnormalities were detected. Subsequently, a cardiac magnetic resonance (CMR) showed a mass in the LA, iso-intense on T1 images (Figure 1C) and with heterogeneous enhancement on late gadolinium (Figure 1D). It was unable to identify a peduncle or clearly identify the site of attachment.

No opacification of the mass was found by angiography. Cardiac computed tomographic scan (CT) also found a mass in the left atrium close to the RIPV but again it failed to characterize its attachment. A transesophageal echocardiography was then performed. It demonstrated the presence of a mobile mass, protruding into the RIPV, without being able to correctly identify its origin. Careful assessment did not reveal other masses in LA and LA appendage. Further 3D acquisition was performed (Figure 2D). 
By using $2 \mathrm{D}$ cutting planes on the 3D volume, the mass was sectioned in the different planes (Figure $2 \mathrm{~A}-\mathrm{C}$ ); by the blue plane of section (panel $\mathrm{C}$ ) we were able to identify the attachment of the mass (panel C, white arrow) as a tiny peduncle (P) to the atrial floor at the base of the IAS close to the opening of RIPV. Surgical resection revealed reddish jelly mass (size $3 \mathrm{~cm} \times 3 \mathrm{~cm}$ ) with soft consistency attached to the atrial floor at the base of the IAS close to the RIPV (Figure 3, arrow). Pathological evaluation confirmed the diagnosis of myxoma. After two years follow up, the patient is doing well without any complication and no recurrence of the tumor.

\section{Discussion}

Cardiac tumors can be divided in primary and metastatic. Most true cardiac tumors are secondary. Primary cardiac tumors are mostly benign and have a good prognosis, whereas malignant primary cardiac tumors are most commonly sarcomas and have a poor prognosis. In adult patients the tumors arising in the left atrium and on the mitral valve are myxomas, papillary fibroelastomas (PFE), lipomas, sarcomas and metastatic tumors [1-5]. Anatomical characteristics of the tumors such as location, mobility, attachment and appearance can help determine whether a mass is benign or malignant. According to the morphologic features, the cardiac tumors in left atrium can be divided in pedunculated and sessile; the myxoma and the PFE are mainly pedunculated while the sarcomas, the lipomas and the secondary tumors are sessile; dealing with the location of the peduncle, the myxoma are mainly attached to the mid portion of the atrial septum while the PFE arise mainly from the valves. Other pedunculated mass arising in the left atrium are the vegetations but they have smaller size except than the fungal and are more frequently attached to the valves. Clots have broader attachment and are mostly located in the LA appendage or on the roof of the LA. Therefore, the detection of a mass attached by a peduncle to the mid-portion of interatrial septum easily lead to the diagnosis of cardiac myxoma [3-7]. Transesophageal and transthoracic echocardiography plays a pivotal role in the imaging [4-7]. If the above anatomical findings are not detected, the differential diagnosis with other cardiac masses becomes more difficult. In this scenario CMR, CT scan and angiography [7,8] can be useful to better delineate the anatomical features and the vascularization of the mass. Three-dimensional echocardiography permits either the anatomical imaging of masses and the selection of unique 2D section planes [5,8-11], which are extremely useful in the assessment of their morphology.

Our case was atypical for many reasons, that could potentially lead to a misdiagnosis of this kind of tumor: $i$ ) the location close to right inferior PV could lead to take into consideration a metastatic tumor coming from the pulmonary vein (PV); ii) the site of attachment at the base of interatrial septum close to the inferior PV is uncommon in the myxomas as they usually originate from the region of the fossa ovalis; iii) the tiny and short peduncle was difficult to detect; iv) the mobility of the mass flipping over the origin of the PV raised the suspicion of a metastatic tumor; v) the surgical appearance, described as red jelly, was uncommon and only at pathology the diagnosis could be confirmed.

Even though a multimodality imaging was performed, the suspicion of myxoma arose at three-dimensional echocardiography that was the only technique able to identify the peduncle and the attachment. In fact, neither the transthoracic nor the transesophageal echocardiography or the CMR were able to identify the site of attachment and the

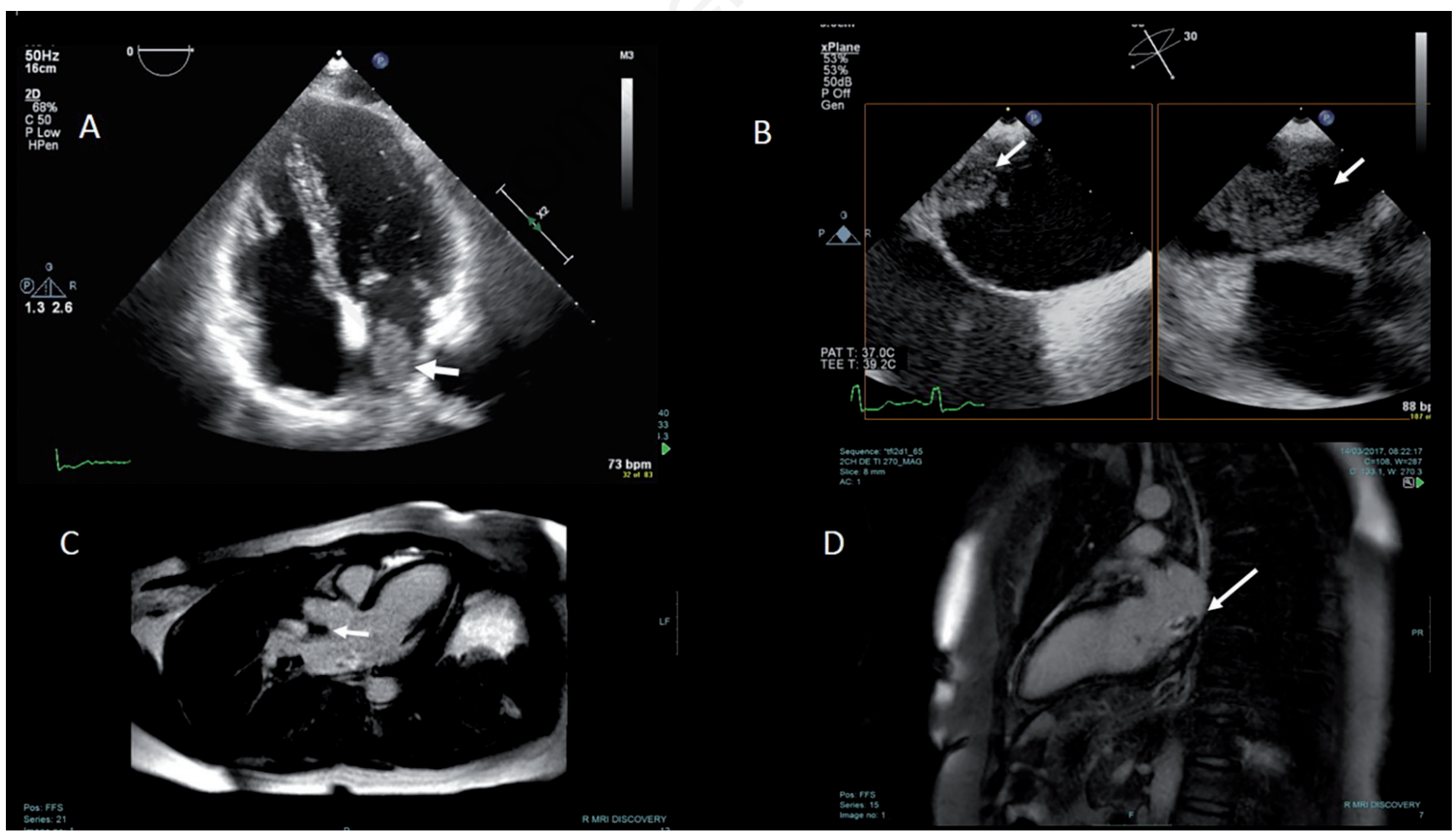

Figure 1. A) Transthoracic echocardiography; 4-chamber view; presence of a mass into the left atrium (white arrow. B) Transesophageal echocardiography, $x$-plane at the level of interatrial septum, LA mass (white arrow). C) CMR showed a mass in the LA, isointense on T1 images (white arrow). D) CMR heterogeneous enhancement on late gadolinium (white arrow). 

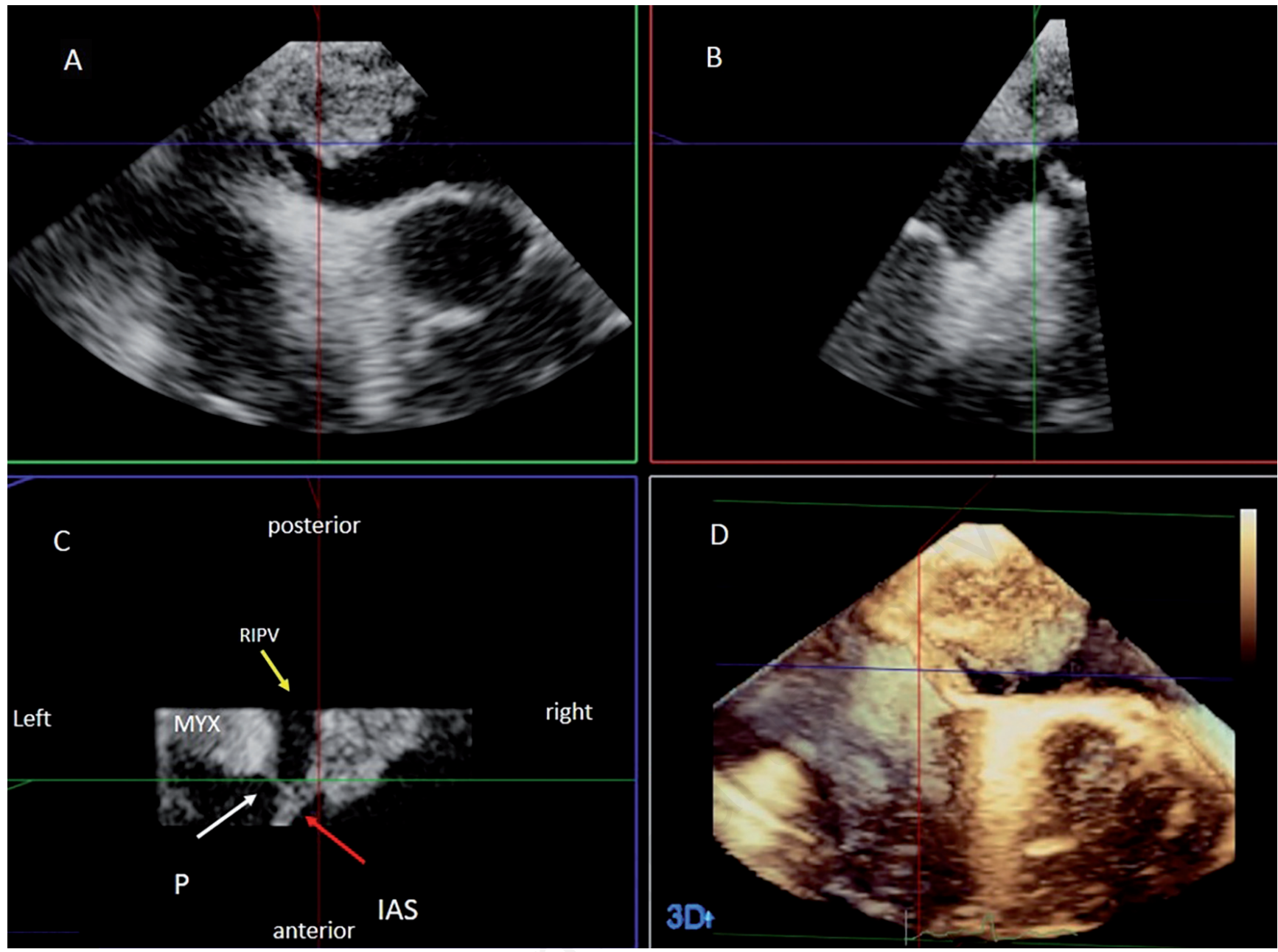

Figure 2. A-C) Three-dimensional echocardiography. C) Identification of the attachment of the tumor by a short and tiny peduncle to the base of IAS (arrow). D) 3DE volume sectioned in different 2D planes of the space.

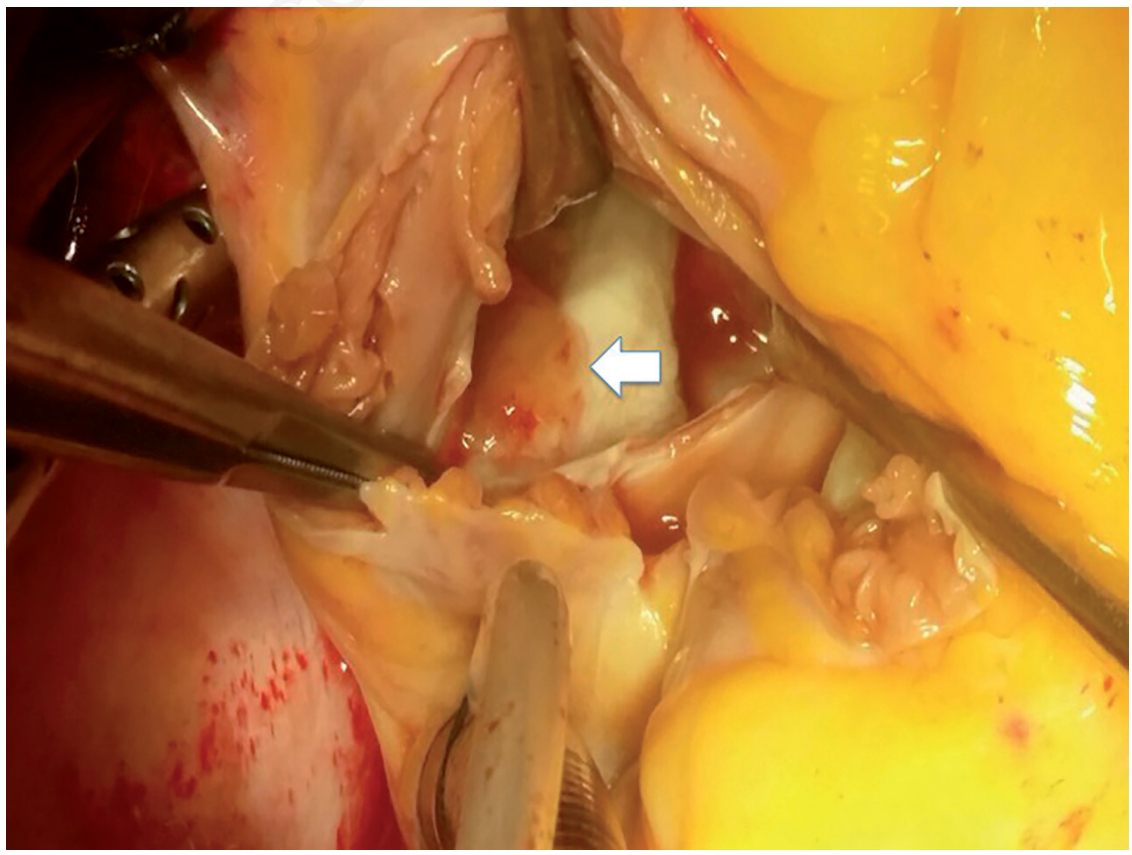

Figure 3. Intraoperative surgical specimen; red jelly mass (white arrow). 
peduncle even with careful revision by experienced operators after the 3D diagnosis; CMR could only confirm the presence of vascularization by the late enhancement and exclude a clot. In order to detect the vascular supply of the mass and in consideration of the risk factors for coronary artery disease, we performed a coronary angiography which did not reveal the presence of neovascularization of the mass. Only the use of the 2D planes generated by electronically sectioning the 3D volume, could correctly identify the attachment and the peduncle of the myxoma.

\section{Conclusions}

Although the ability of 3DE to delineate the site of attachment of cardiac tumors is well known, it was never reported neither in comparison with other diagnostic techniques nor in this unusual location; this case highlights the diagnostic ability of the 3DE and of 2D cutting planes obtained from 3DE in this challenging scenario.

\section{References}

1. Reynen K. Cardiac myxomas. N Engl J Med 1995;333:1610-7.

2. Meng Q, Lai H, Lima $\mathbf{J}$ et al. Echocardiographic and pathologic characteristics of primary cardiac tumors: a study of 149 cases. Int J Cardiol 2002;84:69-75.
3. Espinola-Zavaleta N, Delgado-Barriga J, Soto-Abraham MV. et al. Cardiac benign tumors: echocardiography and computed tomography findings in two cases with histopathologic correlation. Int Canc Conf J 2013;2:82-8.

4. Peters PJ, Reinhardt S. The echocardiographic evaluation of intracardiac masses: A review. J Am Soc Echocardiogr 2006;19:230-40.

5. Mankad R, Herrmann J. Cardiac tumors: echo assessment. Echo Res Pract 2016;3:R65-77.

6. Müller S, Feuchtner G, Bonatti J, et al. Value of transesophageal 3D echocardiography as an adjunct to conventional 2D imaging in preoperative evaluation of cardiac masses. Echocardiography 2008;25:624-31.

7. Mercado-Guzman MP, Meléndez-Ramírez G, Castillo-Castellon F, Kimura-Hayama E. Evaluation of cardiac tumors by multidetector computed tomography and magnetic resonance imaging. Arch Cardiol Mex 2016;86:335-49.

8. Omar HR. The value of coronary angiography in the work-up of atrial myxomas. Herz 2015;40:442-6.

9. Lang RM, Badano LP, Tsang W, et al. EAE/ASE recommendations for image acquisition and display using three-dimensional echocardiography. Eur Heart J Cardiovasc Imaging 2012;13:1-46.

10. Faletra FF, De Castro S, Pandian NG, et al. Atlas of real time 3D transesophageal echocardiography. Dodrecht, Springer: 2010.

11. Espinola-Zavaleta N, Lozoya-Del Rosal JJ, Colin-Lizalde L, LupiHerrera E. Left atrial cardiac myxoma. Two unusual cases studied by 3D echocardiography. BMJ Case Rep 2014;pii:bcr2014205938. 